\title{
一种有效合成 3-芳基-4-氟烷基-2-噁唑烷酮的方法
}

\author{
杨 波 ${ }^{a}$ 赵 敏 ${ }^{a}$ 方 向 ${ }^{a}$ 杨雪艳*,a 吴范宏*,a,b \\ ( ${ }^{a}$ 华东理工大学化学与分子工程学院 上海 200237) \\ ${ }^{b}$ 上海应用技术学院化学与环境工程学院 上海 201418)
}

\begin{abstract}
摘要 报道了一种方便有效地合成 3-芳基-4-氟烷基-2-吘唑烷酮的方法. 用芳基胺作为起始原料通过与氯甲酸烯丙基 酯反应以 $95.8 \% \sim 99.5 \%$ 的收率得到芳基氨基甲酸烯丙基酯. 芳基氨基甲酸烯丙基酯与氟烷基碘在乙腈和水的混合液 中由连二亚硫酸钠引发在室温下发生自由基加成反应得到氟烷基化的加成产物. 加成产物在碱性条件下发生分子内的 $\mathrm{N}$-环合反应得到 3-芳基-4-氟烷基-2-噁唑烷酮. 整个反应都在室温下进行, 并且没有用到光气合成了 3-芳基-4-氟烷基2-嘒唑烷酮，该方法具有原料易得，条件温和，绿色环保的优点.
\end{abstract}

关键词 2-噁唑烷酮; 氟烷基化; 合成; N-环合

\section{A Convenient Synthesis of 3-Aryl-4-fluoroalkyl-2-oxazolidinone}

\author{
${\text { Yang, } \mathrm{Bo}^{a} \quad \text { Zhao, Min }^{a} \quad \text { Fang, Xiang }}^{a} \quad$ Yang, Xueyan*,a $^{*}$ Wu, Fanhong ${ }^{*, a, b}$ \\ ( ${ }^{a}$ School of Chemistry and Molecular Enineering, School of Chemistry and Molecular Enineering, \\ East China University of Science and Technology, Shanghai 200237) \\ $\left({ }^{b}\right.$ School of Chemical and Environmental Engineering, Shanghai Institute of Technology, Shanghai 200235)
}

\begin{abstract}
An efficient and convenient protocol for the synthesis of 3-aryl-4-fluoroalkyl-2-oxazolidinones is described. The allyl arylcarbamates were obtained from the corresponding aromatic amine and chloroformate allyl ester in high yields. The reaction of allyl arylcarbamates with fluoroalkyl iodide initiated by sodium dithionite in aqueous acetonitrile afforded adducts, which underwent cyclization in the presence of sodium hydride to give corresponding 3-aryl-4-fluoroalkyl-2-oxazolidinone in middle yield. All reactions were carried out in room temperature without phosgene to obtain 3-aryl-4-fluoroalkyl2-oxazolidinone. The advantages of this method are that the raw material is easy to get, the operation is simple, it is green and environmental-protection.
\end{abstract}

Keywords oxazolidin-2-ones; fluorinated alkyl; synthesis; $N$-cyclizaion

噁唑烷酮类化合物是一类非常有用的有机化合物, 它们广泛应用于医药、材料和有机合成领域 ${ }^{[1 \sim 4]}$. 在医 药上含有噁唑烷酮结构的化合物已经发现具有优良的 抗惊厥 ${ }^{[5]}$ 和抗菌活性 ${ }^{[6]}$, 特别是在抗菌活性方面, 利奈 唑胺已经作为新一代抗菌药应用于临床治疗各种病菌 感染并且对耐甲氧西林的金黄色葡萄球菌有效 ${ }^{[7 ~ 9]}$. 在 材料领域, 含有噁唑烷酮杂环的材料往往具有耐高温的 特性 ${ }^{[10]}$. 在不对称合成上手性噁唑烷酮还是一种很有 效的诱导辅基 ${ }^{[11 \sim 14]}$.

在有机化合物中引入氟原子往往会极大的改变化 合物的物理化学性质及生物活性 ${ }^{[15]}$. 正因为如此在噁 唑烷酮中引入氟原子或者氟烷基基团成为有机氟化学
工作者们追求的目标. 现有的合成含氟噁唑烷酮的方法 包括亲电氟试剂和啞唑烷酮反应 ${ }^{[16]}$, 含氟砌块和噁唑 烷酮反应 ${ }^{[17]}$ 以及电化学的方法 ${ }^{[18]}$ 等.

最近, 我们已经研究了乙氧基羰基/氨基甲酸烯丙 基酯和氟烷基碘由偶氮二异丁腈(AIBN)引发再经过 $N$ 环合得到含氟烷基的噁唑烷酮的反应，其缺点就是反应 温度太高, 并且对苯基氨基甲酸烯丙基酯只得到痕量的 产物 ${ }^{[19]}$. 为了进一步拓展研究的适用范围, 我们合成了 3-芳基-4-氟烷基-2-噁唑烷酮.

\section{1 结果与讨论}

为了进行我们的研究，首先合成了一系列的芳基氨

* E-mail: yxy@ecust.edu.cn,wfh@ecust.edu.cn

Received October 18, 2012; revised January 21, 2013; published online January 25, 2013.

Project supported by the National Natural Science Foundation of China (Nos. 20972050, 21172148).

国家自然科学基金(Nos. 20972050, 21172148). 
基甲酸烯丙基酯. 按照文献[20]报道的方法很容易就以 95.8\% 99.5\%的收率得到了一系列的芳基氨基甲酸烯 丙基酯 $\mathbf{1 a} \sim 1 \mathrm{e}$ (Eq. 1).

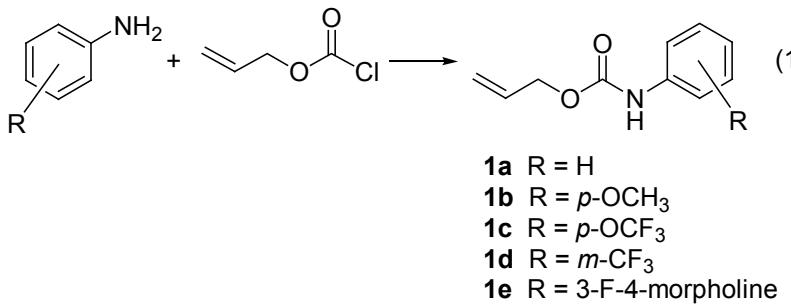

在得到一系列的芳基氨基甲酸烯丙基酯后，我们就 开始研究它们和氟烷基碘的自由基加成反应. 已经报道 过的自由基引发剂有保险粉, AIBN, 过氧化二苯甲酰 (BPO), 金属及其络合物[如 $\mathrm{Cu}, \mathrm{Mg}, \mathrm{Zn} / \mathrm{NiCl}_{6}, \mathrm{CpTiCl}_{2} /$ $\left.\mathrm{Fe}, \mathrm{Pd}\left(\mathrm{PPh}_{3}\right)_{4}\right]$. AIBN, BPO 和金属的引发需要高温的条 件, 并且在以前的工作中发现由 AIBN 引发的反应原料 转化不完全. 综合以上的考虑, 采用保险粉作为研究的 自由基引发剂. 研究发现芳基氨基甲酸烯丙基酯 1a 1e 与氟烷基碘的反应由连二亚硫酸钠在室温下引发就 可以顺利进行, 反应以 $29.3 \% \sim 84.2 \%$ 的收率得到加成 产物 3aa $\sim 3 e c(E q . ~ 2)$. 当氟烷基碘为 $\mathrm{ClCF}_{2} \mathrm{CF}_{2} \mathrm{I}$ (2a)时, 以 $75.3 \% \sim 84.9 \%$ 的收率得到加成产物(表 1, Entries 1 $5)$; 当氟烷基碘为 $\mathrm{Cl}\left(\mathrm{CF}_{2}\right)_{4} \mathrm{I}$ (2b)时以 $45.3 \% \sim 72.3 \%$ 的 收率得到加成产物(表 1, Entries 6 10); 当氟烷基碘为 碘二氟甲基亚磷酸二乙酯时以 $29.3 \% \sim 47.6 \%$ 的收率得 到加成产物(表 1, Entries 11 15). 芳基氨基甲酸酯与氟 烷基碘的反应是一个典型的自由基反应 ${ }^{[21,22]}$, 氟烷基碘 在保险粉的引发下产生自由基与双键发生加成反应得 到加成产物.<smiles>[R][C+](C)[N+](=O)[O-]</smiles>
1a $\sim 1 e$<smiles>[R]CC(I)COC(=O)Nc1cccc([R])c1</smiles>

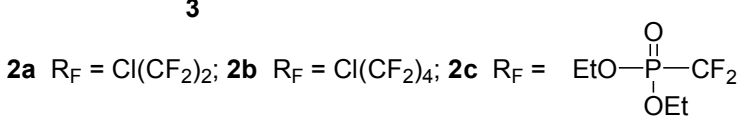

在得到一系列的加成产物后开始对加成产物的分 子内的 N-环合反应进行研究. 为了研究的方便, 我们以 加成产物 3aa 的反应为模板反应对加成产物在碱性条件 下的 N-环合反应进行了研究(Eq. 3). 研究发现碱的强弱 对反应的影响很大, 当碱为弱碱碳酸钾时只有痕量的环 合产物产生, 随着碱性的逐渐增强, 环合产物的收率逐 渐增加, 当碱为强碱正丁基锂时环合产物的收率也开始
表 1 氨基甲酸烯丙基酯(1)与氟烷基碘(2)的自由基加成反应 的结果

Table 1 Results of the radical addition of allyl carbamates (1) with fluoroalkyl iodides (2)

\begin{tabular}{|c|c|c|c|c|}
\hline Entry & Allyl carbamate & Fluoroalkyl iodide & Adduct & Yield/\% \\
\hline 1 & 1a & $2 a$ & 3aa & 75.3 \\
\hline 2 & $1 b$ & $2 a$ & $3 \mathbf{b a}$ & 84.9 \\
\hline 3 & $1 \mathrm{c}$ & $2 \mathbf{a}$ & $3 \mathrm{ca}$ & 82.9 \\
\hline 4 & 1d & $2 \mathbf{a}$ & 3da & 83.2 \\
\hline 5 & $1 \mathrm{e}$ & $2 \mathbf{a}$ & 3ea & 76.1 \\
\hline 6 & 1a & $2 b$ & $3 \mathbf{a b}$ & 63.3 \\
\hline 7 & $1 b$ & $2 b$ & $3 \mathbf{b b}$ & 45.3 \\
\hline 8 & $1 \mathrm{c}$ & $2 b$ & $3 \mathrm{cb}$ & 72.3 \\
\hline 9 & 1d & $2 b$ & $3 d b$ & 45.3 \\
\hline 10 & 1e & $2 b$ & $3 \mathrm{eb}$ & 47.3 \\
\hline 11 & $1 \mathbf{a}$ & $2 c$ & $3 a c$ & 47.6 \\
\hline 12 & $1 b$ & $2 c$ & $3 b c$ & 33.9 \\
\hline 13 & $1 \mathrm{c}$ & $2 c$ & $3 \mathrm{cc}$ & 35.7 \\
\hline 14 & 1d & $2 c$ & $3 d c$ & 29.3 \\
\hline 15 & $1 \mathrm{e}$ & $2 c$ & $3 e c$ & 32.5 \\
\hline
\end{tabular}

下降, 在碱为氢化钠时环合产物 $4 \mathbf{a a}$ 的收率最高(表 2, Entries 1 5). 在此基础上又对反应的溶剂、温度进行了 笁选, 发现在 THF 中, 室温反应收率较高为 $51.4 \%$. 该 反应也同样适用于其他加成产物 $3 \mathbf{a b} \sim \mathbf{3 e c}$, 收率为 $29.3 \% \sim 58.5 \%$ (表 3). 环合产物的收率与碱的强弱和反 应时间有密切的关系，碱性弱，反应时间短时环合反应 不完全，而碱性过强，反应时间过长时环合反应生成的 啞唑烷酮又会发生分解为含氟的氨基醇 ${ }^{[23,24]}$, 在容易分 离的底物 $3 \mathrm{ca}$ 的环合反应中我们不仅以 $58.5 \%$ 的收率得 到了 $4 \mathrm{ca}$, 还得到了 $10.8 \%$ 的含氟氨基醇 $5 \mathrm{ca}(\mathrm{Eq} .4)$.

表 2 化合物 3aa 在不同条件下的 N-环合反应

Table 2 N-cyclization reaction of $\mathbf{3 a a}$ under different conditions

\begin{tabular}{clccc}
\hline Entry & \multicolumn{1}{c}{ Base } & Solvent & Tempt. $/{ }^{\circ} \mathrm{C}$ & Yield $/ \%$ \\
\hline 1 & $\mathrm{~K}_{2} \mathrm{CO}_{3}$ & $\mathrm{THF}$ & 25 & Trace \\
2 & $\mathrm{LiOH}$ & $\mathrm{THF}$ & 25 & 29.2 \\
3 & $\mathrm{NaOH}$ & $\mathrm{THF}$ & 25 & 40.5 \\
4 & $\mathrm{NaH}$ & $\mathrm{THF}$ & 25 & 51.4 \\
5 & $n-\mathrm{BuLi}$ & $\mathrm{THF}$ & 25 & 46.9 \\
6 & $\mathrm{NaH}$ & $\mathrm{CH}_{3} \mathrm{CN}$ & 25 & 13.2 \\
7 & $\mathrm{NaH}$ & $\mathrm{DMF}$ & 25 & 24.3 \\
8 & $\mathrm{NaH}$ & $\mathrm{THF}$ & 0 & 31.3 \\
9 & $\mathrm{NaH}$ & $\mathrm{THF}$ & Reflux & 24.6 \\
\hline
\end{tabular}

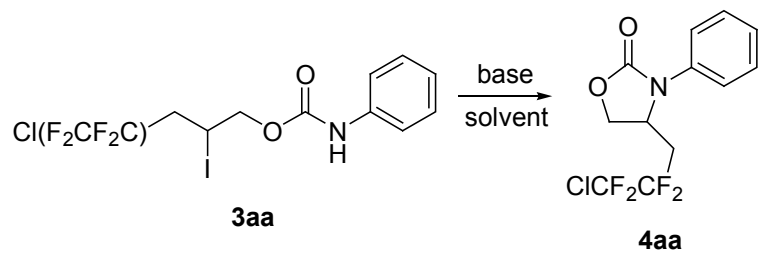




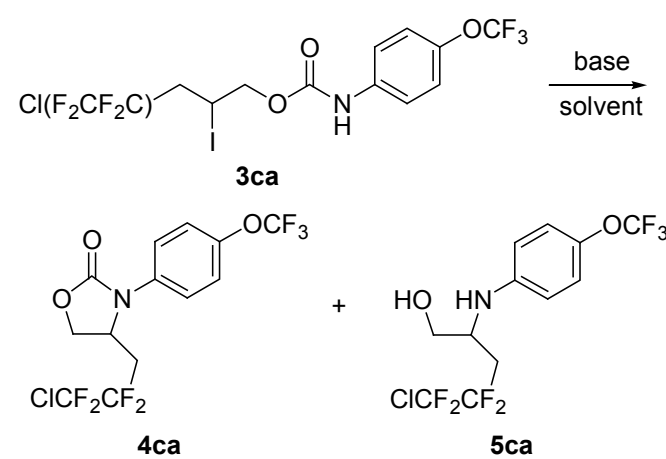

加成产物 3aa 在碱性条件下室温环合得到 4-氟烷基 吘唑烷酮 4aa. 加成产物 $3 a a$ 的分子内 N-环合反应同样 也适用于其他加成产物(Eq. 5), 结果如表 3 所示.<smiles>[R]CC(C)COC(=O)Nc1ccccc1OCCN</smiles><smiles>[R]CC1COC(=O)N1c1ccccc1</smiles>

表 3 化合物 3 在氢化钠的作用下的 N-环合反应的结果 Table 3 Results of N-cyclization reaction of 3 in the presence of $\mathrm{NaH}$

\begin{tabular}{cccc}
\hline Entry & Substrate & Product & Yield/\% \\
\hline 1 & 3aa & 4aa & 51.4 \\
2 & 3ba & 4ba & 52.4 \\
3 & 3ca & 4ca & 58.5 \\
4 & 3da & 4da & 54.2 \\
5 & 3ea & 4ea & 53.4 \\
6 & 3ab & 4ab & 35.2 \\
7 & 3bb & 4bb & 47.3 \\
8 & 3cb & 4cb & 43.2 \\
9 & 3db & 4db & 45.5 \\
10 & 3eb & 4eb & $13.0^{\text {a }}$ \\
11 & 3ac & 4ac & 47.6 \\
12 & 3bc & 4bc & 35.7 \\
13 & 3cc & 4cc & 33.0 \\
14 & 3dc & 4dc & 29.3 \\
15 & 3ec & 4ec & 31.0 \\
\hline
\end{tabular}

${ }^{a}$ Purified by twice recrystallization in ethyl acetate and petroleum ether $(V$ : $V=1: 6$ ).

\section{2 结论}

本文发展了一种以相应的芳基胺为原料通过三步 温和反应制备 4-氟烷基噁唑烷酮的方法. 首先芳基胺和 氯甲酸烯丙基酯在室温下反应以 $95.8 \%$ ～99.5\%的收率 制得芳基氨基甲酸烯丙基酯. 在连二亚硫酸钠的引发 下, 芳基氨基甲酸烯丙基酯与氟烷基碘在乙腈水的混合 液中室温反应 $5 \mathrm{~h}$ 得到收率为 $29.3 \% \sim 84.9 \%$ 的相应的加 成产物. 加成产物在碱存在下室温发生分子内的 N-环 合反应得到 4-氟烷基(包括二氟甲基磷酸二乙酯)腎唑烷 酮. 整个反应都在室温下进行, 并且没有用到光气合成
了 3-芳基-4-氟烷基-2-噁唑烷酮，该方法具有原料易得， 条件温和, 绿色环保的优点.

\section{3 实验部分}

\section{1 仪器与试剂}

质谱仪: Finnigan GC-MS-4021); 核磁共振氢谱仪: Bruker AM 500, ${ }^{1} \mathrm{H}$ NMR (400 MHz), ${ }^{13} \mathrm{C}$ NMR (100.6 $\mathrm{MHz}$ ); 熔点仪: WRS-1B 数字熔点仪(上海精密科学仪 器有限公司); 四氢呋喃，氯甲酸烯丙基酯，碳酸钾，碳 酸钠，分析纯，国药集团化学试剂有限公司; 石油醚、乙 酸乙酯、二氯甲烷、乙腈, 分析纯, 上海凌峰化学试剂 有限公司。

\section{2 芳基氨基甲酸烯丙基酯的合成}

在氩气保护下，向抽烤过的 $100 \mathrm{~mL}$ 的史莱克管中 加入 $40 \mathrm{~mL}$ 四氢呋喃(THF), 苯胺 $(1.84 \mathrm{~g}, 20.00 \mathrm{mmol})$, $\mathrm{K}_{2} \mathrm{CO}_{3}(3.31 \mathrm{~g}, 24.00 \mathrm{mmol}, 1.20$ equiv.)和氯甲酸烯丙基 酯( $2.41 \mathrm{~mL}, 22.00 \mathrm{mmol}, 1.10$ equiv.), 在室温下搅拌反 应 $2 \mathrm{~h}$. 用 $100 \mathrm{ml}$ 二氯甲烷萃取, $50 \mathrm{~mL}$ 饱和 $\mathrm{Na}_{2} \mathrm{CO}_{3}$ 溶 液洗 3 次, 无水硫酸钠干燥. 过滤减压蒸干, 过层析硅 胶柱 $[V$ (石油醚)： $V($ 乙酸乙酯 $)=4 ： 1]$ 得白色固体 $1 \mathbf{a}$ $3.51 \mathrm{~g}$.

苯基氨基甲酸烯丙基酯(1a): 收率 99.5\%. 白色固 体; m.p. 69.3 71.6 ${ }^{\circ} \mathrm{C} ;{ }^{1} \mathrm{H}$ NMR $\left(400 \mathrm{MHz}, \mathrm{CDCl}_{3}\right) \delta$ : $4.70(\mathrm{~d}, J=4.00 \mathrm{~Hz}, 2 \mathrm{H}), 5.27(\mathrm{~d}, J=8.00 \mathrm{~Hz}, 1 \mathrm{H}), 5.38$ (d, $J=13.60 \mathrm{~Hz}, 1 \mathrm{H}), 5.93 \sim 6.04(\mathrm{~m}, 1 \mathrm{H}), 7.08(\mathrm{t}, J=6.20$ $\mathrm{Hz}, 2 \mathrm{H}), 7.17$ (br, 1H), 7.31 (t, $J=6.20 \mathrm{~Hz}, 2 \mathrm{H}), 7.44$ (s, $1 \mathrm{H})$.

4-甲氧基苯基氨基甲酸烯丙基酯(1b)：收率 98.6\%. 白色固体; m.p. 44.0 44.8 ${ }^{\circ} \mathrm{C} ;{ }^{1} \mathrm{H}$ NMR $(400 \mathrm{MHz}$, $\left.\mathrm{CDCl}_{3}\right) \delta: 3.74(\mathrm{~s}, 3 \mathrm{H}), 4.63(\mathrm{~d}, J=5.20 \mathrm{~Hz}, 2 \mathrm{H}), 5.12(\mathrm{~d}$, $J=10.40 \mathrm{~Hz}, 1 \mathrm{H}), 5.32(\mathrm{~d}, J=17.20 \mathrm{~Hz}, 1 \mathrm{H}), 5.89 \sim 6.00$ (m, 1H), $6.81(\mathrm{dd}, J=8.80,2.40 \mathrm{~Hz}, 2 \mathrm{H}), 7.10(\mathrm{br}, 1 \mathrm{H})$, $7.28(\mathrm{~d}, J=6.80 \mathrm{~Hz}, 2 \mathrm{H})$.

4-三氟甲氧基苯基氨基甲酸烯丙基酯(1c): 收率 95.8\%. 白色固体; m.p. $69.3 \sim 71.6{ }^{\circ} \mathrm{C} ;{ }^{1} \mathrm{H}$ NMR (400 $\left.\mathrm{MHz}, \mathrm{CDCl}_{3}\right) \delta: 4.67(\mathrm{~d}, J=6.00 \mathrm{~Hz}, 2 \mathrm{H}), 5.27(\mathrm{~d}, J=$ $10.40 \mathrm{~Hz}, 1 \mathrm{H}), 5.36(\mathrm{~d}, J=17.20 \mathrm{~Hz}, 1 \mathrm{H}), 5.87 \sim 5.97(\mathrm{~m}$, $1 \mathrm{H}), 6.81(\mathrm{br}, 1 \mathrm{H}), 7.16(\mathrm{~d}, J=8.80 \mathrm{~Hz}, 2 \mathrm{H}), 7.41(\mathrm{~d}, J=$ $8.80 \mathrm{~Hz}, 2 \mathrm{H}) ;{ }^{19} \mathrm{~F}$ NMR $\left(376 \mathrm{MHz}, \mathrm{CDCl}_{3}\right) \delta:-58.22$ $(3 \mathrm{~F}, \mathrm{~s})$.

3-三氟甲基苯基氨基甲酸烯丙基酯(1d): 收率 96.5\%. 黄色油状物; ${ }^{1} \mathrm{H} \mathrm{NMR}\left(400 \mathrm{MHz}, \mathrm{CDCl}_{3}\right) \delta: 4.68$ $(\mathrm{d}, J=6.00 \mathrm{~Hz}, 2 \mathrm{H}), 5.26(\mathrm{~d}, J=10.40 \mathrm{~Hz}, 1 \mathrm{H}), 5.35(\mathrm{~d}$ $J=17.20 \mathrm{~Hz}, 1 \mathrm{H}), 5.88 \sim 6.00(\mathrm{~m}, 1 \mathrm{H}), 7.19(\mathrm{br}, 1 \mathrm{H}), 7.30$ (d, $J=8.00 \mathrm{~Hz}, 1 \mathrm{H}), 7.38$ (t, $J=8.00 \mathrm{~Hz}, 1 \mathrm{H}), 7.56$ (d, $J=$ 
$7.60 \mathrm{~Hz}, 1 \mathrm{H}), 7.73(\mathrm{~s}, 1 \mathrm{H}) ;{ }^{19} \mathrm{~F}$ NMR $\left(376 \mathrm{MHz}, \mathrm{CDCl}_{3}\right) \delta$ : $-62.81(\mathrm{~s}, 3 \mathrm{~F})$.

3-氟-4-吗啉基苯基氨基甲酸烯丙基酯(1e): 收率 96.7\%. 棕色固体; m.p. $134.7 \sim 135.8{ }^{\circ} \mathrm{C} ;{ }^{1} \mathrm{H}$ NMR $\left(\mathrm{CDCl}_{3}, 400 \mathrm{MHz}\right) \delta: 3.01(\mathrm{t}, J=3.20 \mathrm{~Hz}, 4 \mathrm{H}), 3.85(\mathrm{t}, J=$ $3.40 \mathrm{~Hz}, 4 \mathrm{H}), 4.63(\mathrm{~d}, J=4.00 \mathrm{~Hz}, 2 \mathrm{H}), 5.23$ (d, $J=8.00$ $\mathrm{Hz}, 1 \mathrm{H}), 5.32(\mathrm{~d}, J=13.60 \mathrm{~Hz}, 1 \mathrm{H}), 5.87 \sim 5.97(\mathrm{~m}, 1 \mathrm{H})$, 6.84 (t, $J=7.20 \mathrm{~Hz}, 1 \mathrm{H}), 6.99$ (d, $J=6.40 \mathrm{~Hz}, 1 \mathrm{H}), 7.15$ (br, 1H), 7.27 (t, $J=5.40 \mathrm{~Hz}, 1 \mathrm{H}) ;{ }^{19} \mathrm{~F}$ NMR $\left(\mathrm{CDCl}_{3}, 376\right.$ MHz) $\delta:-121.22(\mathrm{~s}, 1 \mathrm{~F})$.

\section{3 芳基氨基甲酸(3-氟烷基-2-碘丙基)酯的合成}

将氨基甲酸烯丙基酯(1a) $(0.89 \mathrm{~g}, 5.00 \mathrm{mmol})$, $\mathrm{ClCF}_{2} \mathrm{CF}_{2} \mathrm{I}$ (2a) $(1.97 \mathrm{~g}, 7.50 \mathrm{mmol})$ 和 $\mathrm{MeCN}-\mathrm{H}_{2} \mathrm{O}(V$ : $V=4: 1,25 \mathrm{~mL})$ 加入到 $50 \mathrm{~mL}$ 单口瓶中, 搅拌溶解. 将 $\mathrm{Na}_{2} \mathrm{~S}_{2} \mathrm{O}_{4}(1.31 \mathrm{~g}, 7.50 \mathrm{mmol})$ 和 $\mathrm{NaHCO}_{3}(0.63 \mathrm{~g}, 7.50$ $\mathrm{mmol}$ )加入到反应液中, 室温搅拌反应 $5 \mathrm{~h}$. 反应完全后 加入 $50 \mathrm{~mL}$ 水, 用乙酸乙酯萃取 $(50 \mathrm{~mL} \times 3)$, 无水硫酸 钠干燥, 抽滤, 减压蒸干, 过层析硅胶柱[ $V$ (石油醚) : $V($ 乙酸乙酯 $)=4: 1$ ] 得白色固体 3aa, $1.23 \mathrm{~g}$.

苯基氨基甲酸(5-氯-4,4,5,5-四氟-2-碘戊基)酯(3aa): 收率 73.3\%. 白色固体; m.p. $75.8 \sim 76.9{ }^{\circ} \mathrm{C} ;{ }^{1} \mathrm{H}$ NMR $\left(\mathrm{CDCl}_{3}, 400 \mathrm{MHz}\right) \delta: 2.75 \sim 3.04(\mathrm{~m}, 2 \mathrm{H}), 4.32 \sim 4.51(\mathrm{~m}$, $3 \mathrm{H}), 6.95(\mathrm{br}, 1 \mathrm{H}), 7.10(\mathrm{t}, J=7.20 \mathrm{~Hz}, 1 \mathrm{H}), 7.32(\mathrm{t}, J=$ $8.00 \mathrm{~Hz}, 2 \mathrm{H}), 7.41(\mathrm{~d}, J=7.60 \mathrm{~Hz}, 2 \mathrm{H}) ;{ }^{19} \mathrm{~F}$ NMR $\left(\mathrm{CDCl}_{3}\right.$, $376 \mathrm{MHz}) \delta:-114.50 \sim-112.00(\mathrm{~m}, 2 \mathrm{~F}),-71.80 \sim$ $-71.45(\mathrm{~m}, 2 \mathrm{~F})$.

苯基氨基甲酸(7-氯-4,4,5,5,6,6,7,7-八氟-2-碘庚基) 酯(3ab): 收率 63.3\%. 白色固体; m.p. 77.7 79.1 ${ }^{\circ} \mathrm{C} ;{ }^{1} \mathrm{H}$ NMR $\left(\mathrm{CDCl}_{3}, 400 \mathrm{MHz}\right) \delta: 2.70 \sim 3.00(\mathrm{~m}, 2 \mathrm{H}), 4.35 \sim$ 4.50 (m, 3H), 6.84 (br, 1H), 7.10 (t, $J=7.40 \mathrm{~Hz}, 1 \mathrm{H}), 7.32$ (t, $J=7.80 \mathrm{~Hz}, 2 \mathrm{H}), 7.40(\mathrm{~d}, J=7.60 \mathrm{~Hz}, 2 \mathrm{H}) ;{ }^{19} \mathrm{~F}$ NMR $\left(\mathrm{CDCl}_{3}, 376 \mathrm{MHz}\right) \delta$ : $-123.00 \sim-122.80(\mathrm{~m}, 2 \mathrm{~F})$, $-119.02 \sim-119.71(\mathrm{~m}, 2 \mathrm{~F}),-114.10 \sim-112.40(\mathrm{~m}$, $2 \mathrm{~F}),-68.50 \sim-67.80$ (m, 2F).

苯基氨基甲酸(4-亚磷酸二乙酯基-4,4,-二氟-2-碘丁 基)酯(3ac): 收率 47.6\%. 粘稠液体; ${ }^{1} \mathrm{H} \mathrm{NMR}\left(\mathrm{CDCl}_{3}\right.$, $400 \mathrm{MHz}) \delta: 1.36(\mathrm{t}, J=7.00 \mathrm{~Hz}, 6 \mathrm{H}), 2.06 \sim 2.29(\mathrm{~m}$, $2 \mathrm{H}), 4.02 \sim 4.46(\mathrm{~m}, 7 \mathrm{H}), 7.03(\mathrm{t}, J=7.20 \mathrm{~Hz}, 1 \mathrm{H}), 7.22 \sim$ $7.31(\mathrm{~m}, 2 \mathrm{H}), 7.43(\mathrm{~d}, J=7.80 \mathrm{~Hz}, 2 \mathrm{H}), 7.54(\mathrm{br}, 1 \mathrm{H}) ;{ }^{19} \mathrm{~F}$ NMR $\left(\mathrm{CDCl}_{3}, 376 \mathrm{MHz}\right) \delta:-112.17$ (dd, $J_{\mathrm{F}-\mathrm{F}}=303.06$ $\left.\mathrm{Hz}, J_{\mathrm{F}-\mathrm{P}}=105.28 \mathrm{~Hz}, 1 \mathrm{~F}\right),-108.76\left(J_{\mathrm{F}-\mathrm{F}}=302.68 \mathrm{~Hz}\right.$, $\left.J_{\mathrm{F}-\mathrm{P}}=101.14 \mathrm{~Hz}, 1 \mathrm{~F}\right)$.

4-甲氧基苯基氨基甲酸 5-氯-4,4,5,5-四氟-2-碘戊基) 酯(3ba): 收率 84.2\%. 白色固体; m.p. 51.5 52.5 ${ }^{\circ} \mathrm{C} ;{ }^{1} \mathrm{H}$ NMR $\left(\mathrm{CDCl}_{3}, 400 \mathrm{MHz}\right) \delta: 2.70 \sim 3.00(\mathrm{~m}, 2 \mathrm{H}), 3.78(\mathrm{~s}$,
$3 \mathrm{H}), 4.34 \sim 4.48(\mathrm{~m}, 3 \mathrm{H}), 6.78$ (br, $1 \mathrm{H}), 6.86(\mathrm{~d}, J=8.80$ $\mathrm{Hz}, 2 \mathrm{H}), 7.30(\mathrm{~d}, J=7.20 \mathrm{~Hz}, 2 \mathrm{H}) ;{ }^{19} \mathrm{~F} \mathrm{NMR}\left(\mathrm{CDCl}_{3}, 376\right.$ $\mathrm{MHz}) \delta:-114.40 \sim-112.20(\mathrm{~m}, 2 \mathrm{~F}),-71.60 \sim$ $-71.80(\mathrm{~m}, 2 \mathrm{~F})$.

4-甲氧基苯基氨基甲酸(7-氯-4,4,5,5,6,6,7,7-八氟-2碘庚基)酯(3bb): 收率 45.3\%. 白色固体; m.p. 40.9 $45.3{ }^{\circ} \mathrm{C} ;{ }^{1} \mathrm{H}$ NMR $\left(\mathrm{CDCl}_{3}, 400 \mathrm{MHz}\right) \delta: 2.70 \sim 3.00(\mathrm{~m}$, $2 \mathrm{H}), 3.79(\mathrm{~s}, 3 \mathrm{H}), 4.34 \sim 4.50(\mathrm{~m}, 3 \mathrm{H}), 6.73(\mathrm{br}, 1 \mathrm{H}), 6.86$ (d, $J=9.20 \mathrm{~Hz}, 2 \mathrm{H}), 7.30(\mathrm{~d}, J=7.20 \mathrm{~Hz}, 2 \mathrm{H}) ;{ }^{19} \mathrm{~F}$ NMR $\left(\mathrm{CDCl}_{3}, 376 \mathrm{MHz}\right) \delta:-123.30 \sim-122.26(\mathrm{~m}, 2 \mathrm{~F})$, $-119.97 \sim-120.00(\mathrm{~m}, 2 \mathrm{~F}),-112.20 \sim-113.00(\mathrm{~m}$, $2 \mathrm{~F}),-68.20 \sim-68.00(\mathrm{~m}, 2 \mathrm{~F})$.

4-甲氧基苯基氨基甲酸(4-亚磷酸二乙酯基-4,4,-二 氟-2-碘丁基)酯(3bc): 收率 33.9\%. 粘稠液体; ${ }^{1} \mathrm{H}$ NMR $\left(\mathrm{CDCl}_{3}, 400 \mathrm{MHz}\right) \delta: 1.39(\mathrm{t}, J=7.00 \mathrm{~Hz}, 6 \mathrm{H}), 2.72 \sim 3.04$ (m, $2 \mathrm{H}), 3.79$ (s, 3H), 4.24 4.32 (m, 4H), 4.34 4.44 (m, $2 \mathrm{H}), 4.50 \sim 4.58(\mathrm{~m}, 1 \mathrm{H}), 6.86(\mathrm{~d}, J=9.20 \mathrm{~Hz}, 2 \mathrm{H}), 6.90$ (br, 1H) 7.32 (d, $J=8.00 \mathrm{~Hz}, 2 \mathrm{H}) ;{ }^{19} \mathrm{~F}$ NMR $\left(\mathrm{CDCl}_{3}, 376\right.$ MHz) $\delta:-112.23\left(\mathrm{dd}, J_{\mathrm{F}-\mathrm{F}}=297.42 \mathrm{~Hz}, J_{\mathrm{F}-\mathrm{P}}=103.78 \mathrm{~Hz}\right.$, $1 \mathrm{~F}),-110.69\left(\mathrm{dd}, J_{\mathrm{F}-\mathrm{F}}=297.79 \mathrm{~Hz}, J_{\mathrm{F}-\mathrm{P}}=104.90 \mathrm{~Hz}, 1 \mathrm{~F}\right)$.

4-三氟甲氧基苯基氨基甲酸 (5-氯-4,4,5,5-四氟-2-碘 戊基)酯(3ca): 收率 82.9\%. 白色固体; m.p. 51.4 53.6 ${ }^{\circ} \mathrm{C}$; ${ }^{1} \mathrm{H}$ NMR $\left(\mathrm{CDCl}_{3}, 400 \mathrm{MHz}\right) \delta: 2.73 \sim 3.01(\mathrm{~m}, 2 \mathrm{H})$, $4.36 \sim 4.52$ (m, 3H), 7.09 (br, 1H), $7.17(\mathrm{~d}, J=8.80 \mathrm{~Hz}$, $2 \mathrm{H}), 7.44(\mathrm{~d}, J=8.80 \mathrm{~Hz}, 2 \mathrm{H}) ;{ }^{19} \mathrm{~F}$ NMR $\left(\mathrm{CDCl}_{3}, 376\right.$ $\mathrm{MHz}) \delta:-114.40 \sim-111.80(\mathrm{~m}, 2 \mathrm{~F}),-71.50 \sim$ $-71.90(\mathrm{~m}, 2 \mathrm{~F}),-58.22(\mathrm{~s}, 3 \mathrm{~F})$.

4-三氟甲氧基苯基氨基甲酸(7-氯-4,4,5,5,6,6,7,7-八 氟-2-硔庚基)酯(3cb): 收率 72.3\%. 白色固体; ${ }^{1} \mathrm{H}$ NMR $\left(\mathrm{CDCl}_{3}, 400 \mathrm{MHz}\right) \delta: 2.73 \sim 3.01(\mathrm{~m}, 2 \mathrm{H}), 4.36 \sim 4.52(\mathrm{~m}$, 3H), 6.97 (br, 1H), 7.05 (d, $J=8.80 \mathrm{~Hz}, 2 \mathrm{H}), 7.33$ (d, $J=$ $8.80 \mathrm{~Hz}, 2 \mathrm{H}) ;{ }^{19} \mathrm{~F} \mathrm{NMR}\left(\mathrm{CDCl}_{3}, 376 \mathrm{MHz}\right) \delta:-122.70 \sim$ $-123.30(\mathrm{~m}, 2 \mathrm{~F}), \quad-119.86 \sim-120.80(\mathrm{~m}, 2 \mathrm{~F})$, $-112.0 \sim-115.0(\mathrm{~m}, 2 \mathrm{~F}),-68.00 \sim-68.88(\mathrm{~m}, 2 \mathrm{~F})$, $-58.45(\mathrm{~s}, 3 \mathrm{~F})$.

4-三氟甲氧基苯基氨基甲酸 (4-亚磷酸二乙酯基4,4,-二氟-2-磾丁基)酯(3cc): 收率 35.7\%. 粘稠液体; ${ }^{1} \mathrm{H}$ NMR $\left(\mathrm{CDCl}_{3}, 400 \mathrm{MHz}\right) \delta: 1.30(\mathrm{t}, J=7.00 \mathrm{~Hz}, 6 \mathrm{H})$, $2.60 \sim 3.00(\mathrm{~m}, 2 \mathrm{H}), 4.16 \sim 4.25(\mathrm{~m}, 4 \mathrm{H}), 4.26 \sim 4.38(\mathrm{~m}$, $2 \mathrm{H}), 4.40 \sim 4.50(\mathrm{~m}, 1 \mathrm{H}), 7.07(\mathrm{~d}, J=8.80 \mathrm{~Hz}, 2 \mathrm{H}), 7.39$ $(\mathrm{d}, J=8.80 \mathrm{~Hz}, 2 \mathrm{H}), 7.68(\mathrm{br}, 1 \mathrm{H}) ;{ }^{19} \mathrm{~F}$ NMR $\left(\mathrm{CDCl}_{3}, 376\right.$ MHz) $\delta:-114.00 \sim-108.00(\mathrm{~m}, 2 \mathrm{~F}),-58.23(\mathrm{~s}, 3 \mathrm{~F})$.

3 -三氟甲基苯基氨基甲酸(5-氯-4,4,5,5-四氟-2-碘戊 基)酯(3da): 收率 $83.2 \%$. 粘稠液体; ${ }^{1} \mathrm{H} \mathrm{NMR}\left(\mathrm{CDCl}_{3}\right.$, $400 \mathrm{MHz}) \delta: 2.70 \sim 3.00(\mathrm{~m}, 2 \mathrm{H}), 4.35 \sim 4.55(\mathrm{~m}, 3 \mathrm{H})$, 
$7.21(\mathrm{br}, 1 \mathrm{H}), 7.34$ (d, $J=7.60 \mathrm{~Hz}, 1 \mathrm{H}), 7.42$ (t, $J=7.80$ $\mathrm{Hz}, 1 \mathrm{H}), 7.57$ (t, $J=9.40 \mathrm{~Hz}, 1 \mathrm{H}), 7.73(\mathrm{~s}, 1 \mathrm{H}) ;{ }^{19} \mathrm{~F}$ NMR $\left(\mathrm{CDCl}_{3}, 376 \mathrm{MHz}\right) \delta:-115.00 \sim-111.80(\mathrm{~m}, 2 \mathrm{~F})$, $-71.80 \sim-71.50(\mathrm{~m}, 2 \mathrm{~F}),-62.80(\mathrm{~s}, 3 \mathrm{~F})$.

3 -三氟甲基苯基氨基甲酸(7-氯-4,4,5,5,6,6,7,7-八氟2-碘庚基)酯(3db): 收率 45.3\%。粘稠液体; ${ }^{1} \mathrm{H} \mathrm{NMR}$ $\left(\mathrm{CDCl}_{3}, 400 \mathrm{MHz}\right) \delta: 2.64 \sim 2.90(\mathrm{~m}, 2 \mathrm{H}), 4.27 \sim 4.41(\mathrm{~m}$, 3H), 7.15 (br, 1H), 7.24 (d, $J=7.60 \mathrm{~Hz}, 1 \mathrm{H}), 7.33$ (t, $J=$ $8.00 \mathrm{~Hz}, 1 \mathrm{H}), 7.50(\mathrm{~d}, J=7.60 \mathrm{~Hz}, 1 \mathrm{H}), 7.64(\mathrm{~s}, 1 \mathrm{H}) ;{ }^{19} \mathrm{~F}$ $\operatorname{NMR}\left(\mathrm{CDCl}_{3}, 376 \mathrm{MHz}\right) \delta:-112.80 \sim-123.10(\mathrm{~m}, 2 \mathrm{~F})$, $-120.10 \sim-119.70(\mathrm{~m}, 2 \mathrm{~F}),-115.00 \sim-112.00(\mathrm{~m}$, $2 \mathrm{~F}),-63.30 \sim-68.00(\mathrm{~m}, 2 \mathrm{~F}),-62.91(\mathrm{~s}, 3 \mathrm{~F})$.

3 -三氟甲基苯基氨基甲酸(4-亚磷酸二乙酯基-4,4,二氟-2-碘丁基)酯(2dc): 收率 29.3\%. 粘稠液体; ${ }^{1} \mathrm{H}$ NMR $\left(\mathrm{CDCl}_{3}, 400 \mathrm{MHz}\right) \delta$ : 1.39 (t, $\left.J=7.00 \mathrm{~Hz}, 6 \mathrm{H}\right)$, $2.75 \sim 3.08(\mathrm{~m}, 2 \mathrm{H}), 4.24 \sim 4.32(\mathrm{~m}, 4 \mathrm{H}), 4.37 \sim 4.48(\mathrm{~m}$, 2H), $4.52 \sim 4.61(\mathrm{~m}, 1 \mathrm{H}), 7.32(\mathrm{~d}, J=8.00 \mathrm{~Hz}, 1 \mathrm{H}), 7.42$ (t, $J=8.00 \mathrm{~Hz}, 1 \mathrm{H}), 7.48$ (br, 1H), $7.62(\mathrm{~d}, J=8.00 \mathrm{~Hz}$, $1 \mathrm{H}), 7.75(\mathrm{~s}, 1 \mathrm{H}) ;{ }^{19} \mathrm{~F}$ NMR $\left(\mathrm{CDCl}_{3}, 376 \mathrm{MHz}\right) \delta$ : $-112.09\left(\mathrm{dd}, J_{\mathrm{F}-\mathrm{F}}=298.92 \mathrm{~Hz}, J_{\mathrm{F}-\mathrm{P}}=104.53 \mathrm{~Hz}, 1 \mathrm{~F}\right)$, $-110.55\left(\mathrm{dd}, J_{\mathrm{F}-\mathrm{F}}=298.54 \mathrm{~Hz}, J_{\mathrm{F}-\mathrm{P}}=104.90 \mathrm{~Hz}, 1 \mathrm{~F}\right)$, $-62.78(\mathrm{~s}, 3 \mathrm{~F})$.

3-氟-4-吗啉基苯基氨基甲酸 5-氯-4,4,5,5-四氟-2-碘 戊基)酯(3ea)：收率 76.1\%. 白色固体; m.p. 128.9 130.0 ${ }^{\circ} \mathrm{C} ;{ }^{1} \mathrm{H}$ NMR $\left(\mathrm{CDCl}_{3}, 400 \mathrm{MHz}\right) \delta: 2.76 \sim 3.00(\mathrm{~m}, 2 \mathrm{H})$, $3.05(\mathrm{~s}, 4 \mathrm{H}), 3.88(\mathrm{~s}, 4 \mathrm{H}), 4.32 \sim 4.55(\mathrm{~m}, 3 \mathrm{H}), 6.73(\mathrm{br}$, $1 \mathrm{H}), 6.91(\mathrm{t}, J=6.60 \mathrm{~Hz}, 1 \mathrm{H}), 7.01(\mathrm{~d}, J=6.40 \mathrm{~Hz}, 1 \mathrm{H})$, $7.29(\mathrm{~d}, J=10.8 \mathrm{~Hz}, 1 \mathrm{H}) ;{ }^{19} \mathrm{~F}$ NMR $\left(\mathrm{CDCl}_{3}, 376 \mathrm{MHz}\right) \delta$ : $-120.76(\mathrm{~s}, 1 \mathrm{~F}), \quad-113.50 \sim-111.50$ (m, 2F), $-71.73 \sim-71.65(\mathrm{~m}, 2 \mathrm{~F})$.

3-氟-4-吗啉基苯基氨基甲酸(7-氯-4,4,5,5,6,6,7,7-八 氟-2-碘庚基)酯(3eb): 收率 47.3\%. 白色固体; ${ }^{1} \mathrm{H}$ NMR $\left(\mathrm{CDCl}_{3}, 400 \mathrm{MHz}\right) \delta: 2.74 \sim 2.99(\mathrm{~m}, 2 \mathrm{H}), 3.04$ (t, $J=4.60$ $\mathrm{Hz}, 4 \mathrm{H}), 3.87(\mathrm{t}, J=4.60 \mathrm{~Hz}, 4 \mathrm{H}), 4.36 \sim 4.50(\mathrm{~m}, 3 \mathrm{H})$, 6.88 (t, $J=9.00 \mathrm{~Hz}, 1 \mathrm{H}), 7.03(\mathrm{~d}, J=8.40 \mathrm{~Hz}, 1 \mathrm{H}), 7.30$ $(\mathrm{d}, J=12.00 \mathrm{~Hz}, 1 \mathrm{H}) ;{ }^{19} \mathrm{~F}$ NMR $\left(\mathrm{CDCl}_{3}, 376 \mathrm{MHz}\right) \delta$ : $-122.70 \sim-122.50(\mathrm{~m}, 2 \mathrm{~F}),-119.90 \sim-119.70(\mathrm{~m}$, $2 \mathrm{~F}),-119.07(\mathrm{~s}, 1 \mathrm{~F}),-113.50 \sim-112.00(\mathrm{~m}, 2 \mathrm{~F})$, $-68.20 \sim-68.05(\mathrm{~m}, 2 \mathrm{~F})$.

3-氟-4-吗啉基苯基氨基甲酸(4-亚磷酸二乙酯基4,4,-二氟-2-碘丁基)酯(3ec): 收率 32.5\%. 粘稠液体; ${ }^{1} \mathrm{H}$ NMR $\left(\mathrm{CDCl}_{3}, 400 \mathrm{MHz}\right) \delta: 1.39(\mathrm{t}, J=7.00 \mathrm{~Hz}, 6 \mathrm{H})$, $2.72 \sim 2.99(\mathrm{~m}, 2 \mathrm{H}), 3.03(\mathrm{t}, J=4.60 \mathrm{~Hz}, 4 \mathrm{H}), 3.87(\mathrm{t}, J=$ $4.40 \mathrm{~Hz}, 4 \mathrm{H}), 4.25 \sim 4.34(\mathrm{~m}, 4 \mathrm{H}), 4.35 \sim 4.48(\mathrm{~m}, 2 \mathrm{H})$, $4.50 \sim 4.58(\mathrm{~m}, 1 \mathrm{H}), 6.88(\mathrm{t}, J=9.00 \mathrm{~Hz}, 1 \mathrm{H}), 7.08(\mathrm{~d}, J=$
$8.40 \mathrm{~Hz}, 1 \mathrm{H}), 7.32$ (d, $J=13.20 \mathrm{~Hz}, 1 \mathrm{H}), 7.90(\mathrm{br}, 1 \mathrm{H}) ;{ }^{19} \mathrm{~F}$ NMR $\left(\mathrm{CDCl}_{3}, 376 \mathrm{MHz}\right) \delta$ : $-121.12(\mathrm{~s}, 1 \mathrm{~F}),-112.15$ $\left(\mathrm{dd}, J_{\mathrm{F}-\mathrm{F}}=297.79 \mathrm{~Hz}, J_{\mathrm{F}-\mathrm{P}}=103.78 \mathrm{~Hz}, 1 \mathrm{~F}\right),-110.62(\mathrm{dd}$, $\left.J_{\mathrm{F}-\mathrm{F}}=297.79 \mathrm{~Hz}, J_{\mathrm{F}-\mathrm{P}}=104.53 \mathrm{~Hz}, 1 \mathrm{~F}\right)$.

\subsection{4-氟烷基-3-芳基噁唑烷酮(4ca)和 2-芳基-3-氟烷 基丙醇的合成}

在氩气保护下，向抽烤过的 $50 \mathrm{~mL}$ 的史莱克管中加 入 $20 \mathrm{~mL}$ THF, 加成产物 $3 \mathbf{c a}(1.57 \mathrm{~g}, 3.00 \mathrm{mmol})$ 和氢化 钠( $0.12 \mathrm{~g}, 3.00 \mathrm{mmol}, 1.00$ equiv., $60 \%)$, 室温搅拌反应 2 h. 反应完全后加入 $50 \mathrm{~mL}$ 水, 用乙酸乙酯萃取 $(50$ $\mathrm{mL} \times 3)$, 无水硫酸钠干燥, 抽滤, 减压蒸干, 过层析硅 胶柱 $[V$ (石油醚)： $V$ (乙酸乙酯 $)=7 ： 1$ ]得白色固体 $\mathbf{4 c a}$ $(0.69 \mathrm{~g})$ 和淡黄色液体 $5 \mathbf{c a}(0.12 \mathrm{~g})$.

4-(3-氯-2,2,3,3-四氟丙基-3)-苯基-2-噁唑烷酮(4aa): 收率 51.4\%. 白色固体; m.p. 81.3 81.9 ${ }^{\circ} \mathrm{C} ;{ }^{1} \mathrm{H}$ NMR $\left(\mathrm{CDCl}_{3}, 400 \mathrm{MHz}\right) \delta: 2.28 \sim 2.46(\mathrm{~m}, 1 \mathrm{H}), 2.52 \sim 2.68(\mathrm{~m}$, $1 \mathrm{H}), 4.32(\mathrm{dd}, J=8.80,5.20 \mathrm{~Hz}, 1 \mathrm{H}), 4.70(\mathrm{t}, J=8.6 \mathrm{~Hz}$, $1 \mathrm{H}), 4.80 \sim 4.88(\mathrm{~m}, 1 \mathrm{H}) 7.22 \sim 7.28(\mathrm{~m}, 1 \mathrm{H}), 7.38 \sim 7.48$ $(\mathrm{m}, 4 \mathrm{H}) ;{ }^{19} \mathrm{~F} \mathrm{NMR}\left(\mathrm{CDCl}_{3}, 376 \mathrm{MHz}\right) \delta:-113.5 \sim$ -116.9 (m, 2F), - 71.49, (s, 2F); ${ }^{13} \mathrm{C} \mathrm{NMR}\left(\mathrm{CDCl}_{3}, 107\right.$ MHz) $\delta: 33.14(\mathrm{t}, J=21.94 \mathrm{~Hz}), 50.61,67.47,121.63$, 126.04, 129.70, 135.47, 154.93; HRMS (EI) calcd for $\mathrm{C}_{12} \mathrm{H}_{10} \mathrm{ClF}_{4} \mathrm{NO}_{2}$ 311.0366, found 311.0351.

4-(5-氯-2,2,3,3,4,4,5,5-八氟戊基)-3-苯基-2-噁唑烷 酮(4ab): 收率 35.2\%. 白色固体; m.p. 96.0 100.8 ${ }^{\circ} \mathrm{C}$; ${ }^{1} \mathrm{H}$ NMR $\left(\mathrm{CDCl}_{3}, 400 \mathrm{MHz}\right) \delta: 2.26 \sim 2.48(\mathrm{~m}, 1 \mathrm{H})$, $2.50 \sim 2.68(\mathrm{~m}, 1 \mathrm{H}), 4.32(\mathrm{dd}, J=9.20,5.20 \mathrm{~Hz}, 1 \mathrm{H}), 4.71$ $(\mathrm{t}, J=8.60 \mathrm{~Hz}, 1 \mathrm{H}), 4.80 \sim 4.88(\mathrm{~m}, 1 \mathrm{H}) 7.22 \sim 7.30(\mathrm{~m}$, $2 \mathrm{H}), 7.40 \sim 7.48(\mathrm{~m}, 3 \mathrm{H}) ;{ }^{19} \mathrm{~F} \mathrm{NMR}\left(\mathrm{CDCl}_{3}, 376 \mathrm{MHz}\right) \delta$ : $-112.74 \sim-112.56(\mathrm{~m}, 2 \mathrm{~F}),-120.00 \sim-119.70(\mathrm{~m}$, $2 \mathrm{~F}),-113.00 \sim-112.50(\mathrm{~m}, 2 \mathrm{~F}),-68.17(\mathrm{t}, J=12.78$ $\mathrm{Hz}, 2 \mathrm{~F}) ;{ }^{13} \mathrm{C} \mathrm{NMR}\left(\mathrm{CDCl}_{3}, 107 \mathrm{MHz}\right) \delta: 33.47$ (t, $J=$ $21.77 \mathrm{~Hz}$ ), 50.61, 67.48, 121.63, 126.05, 129.69, 135.44, 154.92; HRMS (EI) calcd for $\mathrm{C}_{14} \mathrm{H}_{10} \mathrm{ClF}_{8} \mathrm{NO}_{2} 411.0272$, found 411.0273 .

4-(磷酸二乙酯基二氟甲基)-甲基-3-苯基-2-噁唑烷 酮(4ac): 收率 47.6\%. 粘稠液体; ${ }^{1} \mathrm{H}$ NMR $\left(\mathrm{CDCl}_{3}, 400\right.$ MHz) $\delta: 1.31 \sim 1.41(\mathrm{~m}, 6 \mathrm{H}), 2.22 \sim 2.41(\mathrm{~m}, 1 \mathrm{H}), 2.49 \sim$ $2.68(\mathrm{~m}, 1 \mathrm{H}), 4.19 \sim 4.36(\mathrm{~m}, 5 \mathrm{H}), 4.66(\mathrm{t}, J=8.60 \mathrm{~Hz}$, $1 \mathrm{H}), 4.85 \sim 4.98(\mathrm{~m}, 1 \mathrm{H}), 7.21(\mathrm{t}, J=7.40 \mathrm{~Hz}, 1 \mathrm{H}), 7.41(\mathrm{t}$, $J=8.00 \mathrm{~Hz}, 2 \mathrm{H}), 7.47(\mathrm{~d}, J=8.80 \mathrm{~Hz}, 2 \mathrm{H}) ;{ }^{19} \mathrm{~F}$ NMR $\left(\mathrm{CDCl}_{3}, 376 \mathrm{MHz}\right) \delta:-108.76\left(\mathrm{dd}, J_{\mathrm{F}-\mathrm{F}}=302.68 \mathrm{~Hz}\right.$, $\left.J_{\mathrm{F}-\mathrm{P}}=101.14 \mathrm{~Hz}, 1 \mathrm{~F}\right),-112.17\left(\mathrm{dd}, J_{\mathrm{F}-\mathrm{F}}=303.06 \mathrm{~Hz}\right.$, $\left.J_{\mathrm{F}-\mathrm{P}}=105.28 \mathrm{~Hz}, 1 \mathrm{~F}\right) ;{ }^{13} \mathrm{C} \mathrm{NMR}\left(\mathrm{CDCl}_{3}, 107 \mathrm{MHz}\right) \delta$ : $16.37,36.20 \sim 36.90(\mathrm{~m}), 50.97,65.03,67.81,121.61$, 
125.60, 129.44, 135.85, 155.20; HRMS (EI) calcd for $\mathrm{C}_{15} \mathrm{H}_{20} \mathrm{~F}_{2} \mathrm{NO}_{5} \mathrm{P} 363.1047$, found 363.1045 .

4-(3-氯-2,2,3,3-四氟丙基)-3-(4-甲氧基苯基)-2-噁唑 烷酮(4ba): 收率 52.4\%. 白色固体; m.p. 93.9 94.5 ${ }^{\circ} \mathrm{C}$; ${ }^{1} \mathrm{H}$ NMR $\left(\mathrm{CDCl}_{3}, 400 \mathrm{MHz}\right) \delta: 2.26 \sim 2.43(\mathrm{~m}, 1 \mathrm{H})$, $2.44 \sim 2.62(\mathrm{~m}, 1 \mathrm{H}), 3.82(\mathrm{~s}, 3 \mathrm{H}), 4.26 \sim 4.32(\mathrm{~m}, 1 \mathrm{H})$, $4.67 \sim 4.78(\mathrm{~m}, 2 \mathrm{H}), 6.96(\mathrm{~d}, J=8.80 \mathrm{~Hz}, 2 \mathrm{H}), 7.29$ (d, $J=$ $8.80,2 \mathrm{H}) ;{ }^{19} \mathrm{~F}$ NMR $\left(\mathrm{CDCl}_{3}, 376 \mathrm{MHz}\right) \delta:-114.00 \sim$ $-110.00(\mathrm{~m}, 2 \mathrm{~F}),-71.63,(\mathrm{~d}, J=7.52 \mathrm{~Hz}, 2 \mathrm{~F}) ;{ }^{13} \mathrm{C} \mathrm{NMR}$ $\left(\mathrm{CDCl}_{3}, 107 \mathrm{MHz}\right) \delta: 33.40(\mathrm{t}, J=22.47 \mathrm{~Hz}), 51.69,55.53$, 67.52, 114.96, 124.55, 128.09, 155.53, 158.06; HRMS (EI) calcd for $\mathrm{C}_{13} \mathrm{H}_{12} \mathrm{ClF}_{4} \mathrm{NO}_{3} 341.0442$, found 341.0441 .

4-(5-氯-2,2,3,3,4,4,5,5-八氟戊基)-3-(4- 甲氧基苯 基)-2-噁唑烷酮 (4bb): 收率 47.3\%. 白色固体; m.p. $109.0 \sim 110.6{ }^{\circ} \mathrm{C} ;{ }^{19} \mathrm{~F}$ NMR $\left(\mathrm{CDCl}_{3}, 376 \mathrm{MHz}\right) \delta: 2.26 \sim$ $2.60(\mathrm{~m}, 2 \mathrm{H}), 3.81$ (s, 3H), $4.27(\mathrm{dd}, J=8.00,4.80 \mathrm{~Hz}$, $1 \mathrm{H}), 4.64 \sim 4.76(\mathrm{~m}, 2 \mathrm{H}), 6.95(\mathrm{~d}, J=8.80 \mathrm{~Hz}, 2 \mathrm{H}), 7.28$ (d, $J=9.20 \mathrm{~Hz}, 2 \mathrm{H}) ;{ }^{19} \mathrm{~F}$ NMR $\left(376 \mathrm{MHz}, \mathrm{CDCl}_{3}\right) \delta$ : $-122.86 \sim-112.64(\mathrm{~m}, 2 \mathrm{~F}),-120.04 \sim-119.84(\mathrm{~m}$, 2F), $-113.57 \sim-111.50(\mathrm{~m}, 2 \mathrm{~F}),-68.35 \sim-68.20(\mathrm{~m}$, 2F); ${ }^{13} \mathrm{C} \mathrm{NMR}\left(\mathrm{CDCl}_{3}, 107 \mathrm{MHz}\right) \delta: 33.61(\mathrm{t}, J=22.09$ Hz), 51.45, 55.43, 67.57, 114.89, 124.67, 128.05, 155.61, 158.07; HRMS (EI) calcd for $\mathrm{C}_{15} \mathrm{H}_{12} \mathrm{ClF}_{8} \mathrm{NO}_{3} 441.0378$, found 441.0381 .

4-(磷酸二乙酯基二氟甲基)-甲基-3-(4-甲氧基苯 基)-2- 腎唑烷酮(4bc): 收率 35.7\%. 粘稠液体; ${ }^{1} \mathrm{H}$ NMR $\left(\mathrm{CDCl}_{3}, 400 \mathrm{MHz}\right) \delta: 1.32 \sim 1.40(\mathrm{~m}, 6 \mathrm{H}), 2.19 \sim 2.38(\mathrm{~m}$, $1 \mathrm{H}), 2.44 \sim 2.62(\mathrm{~m}, 1 \mathrm{H}), 3.80 \sim 3.83(\mathrm{~s}, 3 \mathrm{H}), 4.20 \sim$ $4.32(\mathrm{~m}, 5 \mathrm{H}),, 4.67(\mathrm{t}, J=8.60 \mathrm{~Hz}, 1 \mathrm{H}), 4.75 \sim 4.85(\mathrm{~m}$, $1 \mathrm{H}), 6.94(\mathrm{~d}, J=8.80 \mathrm{~Hz}, 2 \mathrm{H}), 7.33(\mathrm{~d}, J=9.20 \mathrm{~Hz}, 2 \mathrm{H})$; ${ }^{19} \mathrm{~F}$ NMR $\left(\mathrm{CDCl}_{3}, 376 \mathrm{MHz}\right) \delta:-108.90\left(\mathrm{dd}, J_{\mathrm{F}-\mathrm{F}}=\right.$ $\left.302.68 \mathrm{~Hz}, J_{\mathrm{F}-\mathrm{P}}=101.52 \mathrm{~Hz}, 1 \mathrm{~F}\right),-112.06\left(\mathrm{dd}, J_{\mathrm{F}-\mathrm{F}}=\right.$ $\left.302.36 \mathrm{~Hz}, J_{\mathrm{F}-\mathrm{P}}=104.90 \mathrm{~Hz}, 1 \mathrm{~F}\right) ;{ }^{13} \mathrm{C} \mathrm{NMR}\left(\mathrm{CDCl}_{3}, 107\right.$ MHz) $\delta: 16.38,36.40 \sim 37.10(\mathrm{~m}), 51.74,55.54,65.00$, 67.95, 114.75, 124.41, 128.50, 155.76, 157.76; HRMS (EI) calcd for $\mathrm{C}_{16} \mathrm{H}_{22} \mathrm{~F}_{2} \mathrm{NO}_{6} \mathrm{P}$ 393.1193, found 393.1151.

4-(3-氯-2,2,3,3-四氟丙基)-3-(4-三氟甲氧基苯基)-2噁唑烷酮(4ca): 收率 58.5\%. 白色固体; m.p. 82.3 85.8 ${ }^{\circ} \mathrm{C} ;{ }^{1} \mathrm{H}$ NMR $\left(\mathrm{CDCl}_{3}, 400 \mathrm{MHz}\right) \delta$ : $2.32 \sim 2.47(\mathrm{~m}, 1 \mathrm{H})$, $2.48 \sim 2.66(\mathrm{~m}, 1 \mathrm{H}), 4.34(\mathrm{dd}, J=9.20,5.20 \mathrm{~Hz}, 1 \mathrm{H}), 4.71$ (t, $J=8.60 \mathrm{~Hz}, 1 \mathrm{H}), 4.78 \sim 4.88(\mathrm{~m}, 1 \mathrm{H}), 7.29$ (d, $J=8.40$ $\mathrm{Hz}, 2 \mathrm{H}), 7.48(\mathrm{~d}, J=8.80 \mathrm{~Hz}, 2 \mathrm{H}) ;{ }^{19} \mathrm{~F}$ NMR $\left(\mathrm{CDCl}_{3}, 376\right.$ $\mathrm{MHz}) \delta: \quad-113.50 \sim-111.50(\mathrm{~m}, 2 \mathrm{~F}),-71.69 \sim$ $-71.63(\mathrm{~m}, 2 \mathrm{~F}),-58.06$ (s, 3F); ${ }^{13} \mathrm{C} \mathrm{NMR}\left(\mathrm{CDCl}_{3}, 107\right.$ MHz) $\delta: 33.14$ (t, $J=22.42 \mathrm{~Hz}), 50.82,67.48,122.29$,
$122.68,134.22,146.58,154.82$; HRMS (EI) calcd for $\mathrm{C}_{15} \mathrm{H}_{9} \mathrm{ClF}_{7} \mathrm{NO}_{3} 395.0159$, found 395.0156 .

4-(5-氯-2,2,3,3,4,4,5,5-八氟戊基)-3-(4-三氟甲氧基 苯基)-2-噁唑烷酮(4cb)：收率 43.2\%. 白色固体; m.p. $87.0 \sim 89.8{ }^{\circ} \mathrm{C} ;{ }^{1} \mathrm{H}$ NMR $\left(\mathrm{CDCl}_{3}, 400 \mathrm{MHz}\right) \delta: 2.31 \sim 2.69$ (m, 2H), 4.33 (dd, $J=9.20,4.80 \mathrm{~Hz}, 1 \mathrm{H}), 4.72$ (t, $J=8.80$ $\mathrm{Hz}, 1 \mathrm{H}), 4.80 \sim 4.88(\mathrm{~m}, 1 \mathrm{H}), 7.30(\mathrm{~d}, J=8.80 \mathrm{~Hz}, 2 \mathrm{H})$, $7.48(\mathrm{~d}, J=9.20 \mathrm{~Hz}, 2 \mathrm{H}) ;{ }^{19} \mathrm{~F}$ NMR $\left(\mathrm{CDCl}_{3}, 376 \mathrm{MHz}\right) \delta$ : $-123.20 \sim-122.60(\mathrm{~m}, 2 \mathrm{~F}),-119.90 \sim-119.76(\mathrm{~m}$, $2 \mathrm{~F}),-113.00 \sim-112.50(\mathrm{~m}, 2 \mathrm{~F}),-68.26 \sim-68.12(\mathrm{~m}$, $2 \mathrm{~F}),-58.07(\mathrm{~s}, 3 \mathrm{~F}) ;{ }^{13} \mathrm{C}$ NMR $\left(\mathrm{CDCl}_{3}, 107 \mathrm{MHz}\right) \delta$ : $33.43(\mathrm{t}, J=21.83 \mathrm{~Hz}), 50.53,67.50,122.29,122.60$, 134.12, 146.58, 154.73; HRMS (EI) calcd for $\mathrm{C}_{15} \mathrm{H}_{9} \mathrm{Cl}-$ $\mathrm{F}_{11} \mathrm{NO}_{2}$ 495.0095, found 495.0093.

4-(磷酸二乙酯基二氟甲基)-甲基-3-(4-三氟甲氧基 苯基)-2-噁唑烷酮 (4cc)：收率 33.0\%. 粘稠液体; ${ }^{1} \mathrm{H}$ NMR $\left(\mathrm{CDCl}_{3}, 400 \mathrm{MHz}\right) \delta: 1.33 \sim 1.43(\mathrm{~m}, 6 \mathrm{H}), 2.24 \sim$ $2.42(\mathrm{~m}, 1 \mathrm{H}), 2.47 \sim 2.68(\mathrm{~m}, 1 \mathrm{H}), 4.23 \sim 4.37(\mathrm{~m}, 5 \mathrm{H})$, $4.67(\mathrm{t}, J=8.60 \mathrm{~Hz}, 1 \mathrm{H}), 4.85 \sim 4.98(\mathrm{~m}, 1 \mathrm{H}), 7.27(\mathrm{~d}, J=$ $7.60 \mathrm{~Hz}, 2 \mathrm{H}), 7.37(\mathrm{~d}, J=8.80 \mathrm{~Hz}, 2 \mathrm{H}) ;{ }^{19} \mathrm{~F} \mathrm{NMR}\left(\mathrm{CDCl}_{3}\right.$, $376 \mathrm{MHz}) \delta:-108.58\left(\mathrm{dd}, J_{\mathrm{F}-\mathrm{F}}=303.43 \mathrm{~Hz}, J_{\mathrm{F}-\mathrm{P}}=100.77\right.$ $\mathrm{Hz}, 1 \mathrm{~F}),-112.27\left(\mathrm{dd}, J_{\mathrm{F}-\mathrm{F}}=303.43 \mathrm{~Hz}, J_{\mathrm{F}-\mathrm{P}}=105.66 \mathrm{~Hz}\right.$, $1 \mathrm{~F}),-58.10(\mathrm{~s}, 3 \mathrm{~F}) ;{ }^{13} \mathrm{C}$ NMR $\left(\mathrm{CDCl}_{3}, 107 \mathrm{MHz}\right) \delta$ : $16.37,36.00 \sim 37.20(\mathrm{~m}), 50.97,65.03,67.81,121.61$, 125.60, 129.44, 135.85, 155.20; HRMS (EI) calcd for $\mathrm{C}_{16} \mathrm{H}_{19} \mathrm{~F}_{5} \mathrm{NO}_{6} \mathrm{P} 447.0870$, found 447.0872 .

4-(3-氯-2,2,3,3-四氟丙基)-3-(3-三氟甲基苯基)-2-噁 唑烷酮(4da): 收率 54.2\%. 粘稠液体; ${ }^{1} \mathrm{H}$ NMR $\left(\mathrm{CDCl}_{3}\right.$, $400 \mathrm{MHz}) \delta: 2.36 \sim 2.66(\mathrm{~m}, 2 \mathrm{H}), 4.32 \sim 4.38(\mathrm{~m}, 1 \mathrm{H})$, $4.78 \sim 4.88(\mathrm{~m}, 1 \mathrm{H}), 4.72(\mathrm{t}, J=8.60 \mathrm{~Hz}, 1 \mathrm{H}), 7.49(\mathrm{~d}, J=$ $8.00 \mathrm{~Hz}, 1 \mathrm{H}), 7.57(\mathrm{t}, J=8.00 \mathrm{~Hz}, 1 \mathrm{H}), 7.66(\mathrm{~d}, J=8.40$ $\mathrm{Hz}, 1 \mathrm{H}), 7.75(\mathrm{~s}, 1 \mathrm{H}) ;{ }^{19} \mathrm{~F} \mathrm{NMR}\left(\mathrm{CDCl}_{3}, 376 \mathrm{MHz}\right) \delta$ : $113.35 \sim-111.40(\mathrm{~m}, 2 \mathrm{~F}),-71.75$, (s, 2F), $-62.93(\mathrm{~s}$, $3 \mathrm{~F}) ;{ }^{13} \mathrm{C}$ NMR $\left(\mathrm{CDCl}_{3}, 107 \mathrm{MHz}\right) \delta: 33.06(\mathrm{t}, J=22.52$ Hz), 50.49, 67.47, 117.69, 122.25, 123.86, 130.27, 131.94 , 132.27, 136.34, 154.50; HRMS (EI) calcd for $\mathrm{C}_{13} \mathrm{H}_{9} \mathrm{Cl}-$ $\mathrm{F}_{7} \mathrm{NO}_{2} 379.0210$, found 379.0211 .

4-(5-氯-2,2,3,3,4,4,5,5-八氟戊基)-3-(3-三氟甲基苯 基)-2-噁唑烷酮(4db): 收率 45.5\%. 粘稠液体; ${ }^{1} \mathrm{H}$ NMR $\left(\mathrm{CDCl}_{3}, 400 \mathrm{MHz}\right) \delta: 2.36 \sim 2.66(\mathrm{~m}, 2 \mathrm{H}), 4.35$ (dd, $J=$ $9.20,4.80 \mathrm{~Hz}, 1 \mathrm{H}), 4.72(\mathrm{t}, J=8.80 \mathrm{~Hz}, 1 \mathrm{H}), 4.87 \sim 4.96$ (m, 1H), 7.49 (d, $J=7.60 \mathrm{~Hz}, 1 \mathrm{H}), 7.57$ (t, $J=7.80 \mathrm{~Hz}$, $1 \mathrm{H}), 7.65(\mathrm{~d}, J=8.40 \mathrm{~Hz}, 1 \mathrm{H}), 7.76(\mathrm{~s}, 1 \mathrm{H}) ;{ }^{19} \mathrm{~F}$ NMR $\left(\mathrm{CDCl}_{3}, 376 \mathrm{MHz}\right) \delta:-122.85 \sim-122.68(\mathrm{~m}, 2 \mathrm{~F})$, $-119.95 \sim-119.81(\mathrm{~m}, 2 \mathrm{~F}),-113.50 \sim-112.53(\mathrm{~m}$, 
$2 \mathrm{~F}),-68.40 \sim-68.16(\mathrm{~m}, 2 \mathrm{~F}),-63.02(\mathrm{~s}, 3 \mathrm{~F}) ;{ }^{13} \mathrm{C}$ NMR $\left(\mathrm{CDCl}_{3}, 107 \mathrm{MHz}\right) \delta: 33.37(\mathrm{t}, J=22.20 \mathrm{~Hz}), 50.26$, $67.52,117.72,122.24,123.86,124.89,130.26,132.12$, 136.30, 154.49; HRMS (EI) calcd for $\mathrm{C}_{15} \mathrm{H}_{9} \mathrm{ClF}_{11} \mathrm{NO}_{2}$ 479.0146, found 479.0141 .

4-(磷酸二乙酯基二氟甲基)-甲基-3-(3-三氟甲基苯 基)-2-噁唑烷酮(4dc): 收率 29.3\%. 粘稠液体; ${ }^{1} \mathrm{H}$ NMR $\left(\mathrm{CDCl}_{3}, 400 \mathrm{MHz}\right) \delta: 1.34 \sim 1.41(\mathrm{~m}, 6 \mathrm{H}), 2.27 \sim 2.44(\mathrm{~m}$, $1 \mathrm{H}), 2.45 \sim 2.66(\mathrm{~m}, 1 \mathrm{H}), 4.18 \sim 4.42(\mathrm{~m}, 5 \mathrm{H}), 4.68(\mathrm{t}, J=$ $8.80 \mathrm{~Hz}, 1 \mathrm{H}), 4.95 \sim 5.04(\mathrm{~m}, 1 \mathrm{H}), 7.46(\mathrm{~d}, J=8.00 \mathrm{~Hz}$, $1 \mathrm{H}), 7.55(\mathrm{t}, J=8.00 \mathrm{~Hz}, 1 \mathrm{H}), 7.75(\mathrm{~d}, J=8.40 \mathrm{~Hz}, 1 \mathrm{H})$, 7.79 (s, $1 \mathrm{H}) ;{ }^{19} \mathrm{~F}$ NMR $\left(\mathrm{CDCl}_{3}, 376 \mathrm{MHz}\right) \delta$ : -112.29 $\left(\mathrm{dd}, J_{\mathrm{F}-\mathrm{F}}=303.43 \mathrm{~Hz}, J_{\mathrm{F}-\mathrm{P}}=104.90 \mathrm{~Hz}, 1 \mathrm{~F}\right),-108.56(\mathrm{dd}$, $\left.J_{\mathrm{F}-\mathrm{F}}=303.81 \mathrm{~Hz}, J_{\mathrm{F}-\mathrm{P}}=101.14 \mathrm{~Hz}, 1 \mathrm{~F}\right),-62.78(\mathrm{~s}, 3 \mathrm{~F})$; ${ }^{13} \mathrm{C} \mathrm{NMR}\left(\mathrm{CDCl}_{3}, 107 \mathrm{MHz}\right) \delta: 16.26 \sim 16.40(\mathrm{~m}), 29.70$, $36.20 \sim 37.20(\mathrm{~m}), 65.00 \sim 65.50(\mathrm{~m}), 67.86,117.44$, $121.84,124.00,130.06,131.85,136.69,154.77$; HRMS (EI) calcd for $\mathrm{C}_{16} \mathrm{H1}_{9} \mathrm{~F}_{5} \mathrm{NO}_{5} 431.0921$, found 431.0293.

4-(3-氯-2,2,3,3-四氟丙基)-3-(3-氟-4-吗啉基苯基)-2噁唑烷酮 (4ea): 收率 53.4\%. 褐色固体; m.p. 118.3 $119.1{ }^{\circ} \mathrm{C} ;{ }^{1} \mathrm{H}$ NMR $\left(\mathrm{CDCl}_{3}, 400 \mathrm{MHz}\right) \delta: 2.30 \sim 2.61(\mathrm{~m}$, $2 \mathrm{H}), 3.07$ (t, $J=3.40 \mathrm{~Hz}, 4 \mathrm{H}), 3.85(\mathrm{t}, J=3.40 \mathrm{~Hz}, 4 \mathrm{H})$, $4.28(\mathrm{dd}, J=7.20,4.00 \mathrm{~Hz}, 1 \mathrm{H}), 4.66(\mathrm{t}, J=6.80 \mathrm{~Hz}, 1 \mathrm{H})$, $4.69 \sim 4.76(\mathrm{~m}, 1 \mathrm{H}), 6.95(\mathrm{t}, J=7.20 \mathrm{~Hz}, 1 \mathrm{H}), 7.02(\mathrm{~d}, J=$ $6.00 \mathrm{~Hz}, 1 \mathrm{H}), 7.25(J=10.80 \mathrm{~Hz}, 1 \mathrm{H}) ;{ }^{19} \mathrm{~F}$ NMR $\left(\mathrm{CDCl}_{3}\right.$, $376 \mathrm{MHz}) \delta$ : $-119.44(\mathrm{~s}, 1 \mathrm{~F}),-113.35 \sim-111.58(\mathrm{~m}$, $2 \mathrm{~F}),-71.74 \sim-71.66(\mathrm{~m}, 2 \mathrm{~F}) ;{ }^{13} \mathrm{C} \mathrm{NMR}\left(\mathrm{CDCl}_{3}, 107\right.$ $\mathrm{MHz}) \delta: 33.04(\mathrm{t}, J=17.82 \mathrm{~Hz}), 50.73,51.05,66.83$, $67.34,110.96,117.67,119.07,138.03,136.06,154.89$, 156.44; HRMS (EI) calcd for $\mathrm{C}_{16} \mathrm{H}_{16} \mathrm{ClF}_{5} \mathrm{~N}_{2} \mathrm{O}_{3} 414.0770$, found 414.0760 .

4-(5-氯-2,2,3,3,4,4,5,5-八氟戊基)-3-(3-氟-4-吗啉基 苯基)-2-噁唑烷酮(4eb): 收率 13.0\%. 白色固体; m.p. $128.0 \sim 129.7{ }^{\circ} \mathrm{C} ;{ }^{1} \mathrm{H}$ NMR $\left(\mathrm{CDCl}_{3}, 400 \mathrm{MHz}\right) \delta: 2.28 \sim$ $2.46(\mathrm{~m}, 1 \mathrm{H}), 2.48 \sim 2.68(\mathrm{~m}, 1 \mathrm{H}), 3.10(\mathrm{t}, J=4.60 \mathrm{~Hz}$, $4 \mathrm{H}), 3.88(\mathrm{t}, J=4.80 \mathrm{~Hz}, 4 \mathrm{H}), 4.31(\mathrm{dd}, J=8.40,4.40 \mathrm{~Hz}$, $1 \mathrm{H}), 4.65 \sim 4.78(\mathrm{~m}, 2 \mathrm{H}), 6.94 \sim 7.05(\mathrm{~m}, 2 \mathrm{H}), 7.27 \sim 7.32$ $(\mathrm{m}, 1 \mathrm{H}) ;{ }^{19} \mathrm{~F}$ NMR $\left(\mathrm{CDCl}_{3}, 376 \mathrm{MHz}\right) \delta:-112.70 \sim$ -112.51 (m, 2F), $-119.89 \sim-119.71 \quad(\mathrm{~m}, 2 \mathrm{~F})$, -119.07 (s, 1F), $-113.8 \sim 111.8(\mathrm{~m}, 2 \mathrm{~F}),-68.34 \sim$ $-67.74(\mathrm{~m}, 2 \mathrm{~F}) ;{ }^{13} \mathrm{C} \mathrm{NMR}\left(\mathrm{CDCl}_{3}, 107 \mathrm{MHz}\right) \delta: 33.58 \sim$ $33.70(\mathrm{~m}), 50.79,51.05,66.91,67.44,110.62,117.69$, $119.15,121.63,129.91,135.52,138.16,136.06,154.34$, 154.91, 156.80; HRMS (EI) calcd for $\mathrm{C}_{18} \mathrm{H}_{16} \mathrm{ClF}_{9} \mathrm{~N}_{2} \mathrm{O}_{3}$ 514.0706 , found 514.0695 .
4-(磷酸二乙酯基二氟甲基)-甲基-3-(3-氟-4-吗啉基 苯基)-2-噁唑烷酮 (4ec)：收率 31.0\%。粘稠液体; ${ }^{1} \mathrm{H}$ NMR $\left(\mathrm{CDCl}_{3}, 400 \mathrm{MHz}\right) \delta: 1.35 \sim 1.42(\mathrm{~m}, 6 \mathrm{H}), 2.21 \sim$ $2.41(\mathrm{~m}, 1 \mathrm{H}), 2.48 \sim 2.66(\mathrm{~m}, 1 \mathrm{H}), 3.07(\mathrm{t}, J=4.60 \mathrm{~Hz}$, $4 \mathrm{H}), 3.79 \sim 3.90(\mathrm{t}, J=4.60 \mathrm{~Hz}, 4 \mathrm{H}), 4.22 \sim 4.36(\mathrm{~m}, 5 \mathrm{H})$, $4.65(\mathrm{t}, J=8.80 \mathrm{~Hz}, 1 \mathrm{H}), 4.78 \sim 4.87(\mathrm{~m}, 1 \mathrm{H}), 6.96(\mathrm{t}, J=$ $9.20 \mathrm{~Hz}, 1 \mathrm{H}), 7.13(\mathrm{~d}, J=8.80 \mathrm{~Hz}, 1 \mathrm{H}), 7.29 \sim 7.34(\mathrm{~m}$, $1 \mathrm{H}) ;{ }^{19} \mathrm{~F} \mathrm{NMR}\left(\mathrm{CDCl}_{3}, 376 \mathrm{MHz}\right) \delta:-119.71(\mathrm{~s}, 1 \mathrm{~F})$, $-112.13\left(\mathrm{dd}, J_{\mathrm{F}-\mathrm{F}}=303.06 \mathrm{~Hz}, J_{\mathrm{F}-\mathrm{P}}=105.66 \mathrm{~Hz}, 1 \mathrm{~F}\right)$, $-108.61\left(\mathrm{dd}, J_{\mathrm{F}-\mathrm{F}}=302.68 \mathrm{~Hz}, J_{\mathrm{F}-\mathrm{P}}=100.77 \mathrm{~Hz}, 1 \mathrm{~F}\right) ;{ }^{13} \mathrm{C}$ NMR (107 MHz, $\left.\mathrm{CDCl}_{3}\right) \delta: 16.39$ (d, $\left.J=5.67 \mathrm{~Hz}\right), 29.71$, $36.00 \sim 36.80(\mathrm{~m}), 50.80 \sim 51.30(\mathrm{~m}), 64.80 \sim 65.50(\mathrm{~m})$, $66.91,67.82,110.62,117.49,119.11,130.58,137.62$, 155.51, 156.78; HRMS (EI) calcd for $\mathrm{C}_{19} \mathrm{H}_{26} \mathrm{ClF}_{3} \mathrm{~N}_{2} \mathrm{O}_{6}$ 466.1481 , found 466.1483 .

5-氯-4,4,5,5-四氟-2-[4-(三氟甲氧基)苯胺]戊醇 (5ca): 收率 $10.8 \%$. 淡黄色液体; ${ }^{1} \mathrm{H}$ NMR $\left(\mathrm{CDCl}_{3}, 400\right.$ $\mathrm{MHz}) \delta: 2.18 \sim 2.42(\mathrm{~m}, 2 \mathrm{H}), 3.65 \sim 3.71(\mathrm{~m}, 1 \mathrm{H}), 3.76 \sim$ $3.86(\mathrm{~m}, 2 \mathrm{H}), 6.53(\mathrm{~d}, J=9.20 \mathrm{~Hz}, 2 \mathrm{H}), 6.97(\mathrm{~d}, J=8.40$ $\mathrm{Hz}, 2 \mathrm{H}) ;{ }^{19} \mathrm{~F}$ NMR $\left(\mathrm{CDCl}_{3}, 376 \mathrm{MHz}\right) \delta:-113.00 \sim$ $-112.50(\mathrm{~m}, 2 \mathrm{~F}),-71.62 \sim-71.56(\mathrm{~m}, 2 \mathrm{~F}) ;{ }^{13} \mathrm{C} \mathrm{NMR}$ $\left(\mathrm{CDCl}_{3}, 107 \mathrm{MHz}\right) \delta: 31.25(\mathrm{t}, J=23.01 \mathrm{~Hz}), 49.20,63.73$, 113.94, 122.71, 141.19, 144.79; HRMS (EI) calcd for $\mathrm{C}_{12} \mathrm{H}_{11} \mathrm{ClF}_{7} \mathrm{NO}_{2} 369.0367$, found 369.0365 .

\section{References}

[1] Dyen, M. E.; Swern, D. Chem. Rev. 1967, 67, 197.

[2] Tam, W. J. J. Org. Chem. 1986, 51, 2977.

[3] Wouters, J.; Vercauteren, D. P.; Collin, S.; Evrard, G.; Durant, F.; Ducrey, F.; Koenig, J. J.; Jarreau, F. X. Eur. J. Med. Chem. 1992, 27, 939.

[4] Moureau, F.; Wouters, J.; Vercauteren, D. P.; Collin, S.; Evrard, G..; Durant, F.; Ducrey, F.; Koenig, J. J.; Jarreau, F. X. Eur. J. Med. Chem. 1994, 29, 269.

[5] Close, W. J. J. Am. Chem. Soc. 1951, 73, 95.

[6] Kudo, N.; Taniguchi, M.; Furuta, S.; Sato, K.; Endo, T.; Honma, T. J. Agric. Food Chem. 1998, 46, 5305.

[7] Choy, A.; Colbry, N.; Huber, C.; Pamment, M.; Duine, J. V. Org. Process Res. Dev. 1998, 12, 884.

[8] Khalaj, A.; Nakhjiri, M.; Negahbani, A. S.; Samadizadeh, M.; Firoozpour, L.; Rajabalian, S.; Samadi, N.; Faramarzi, M. A.; Adibpour, N.; Shafiee, A.; Foroumadi, A. Eur. J. Med. Chem. 2011, 46, 65.

[9] Selvakumar, N.; Srinivas, D.; Khera, M. K.; Kumar, M. S.; Mamidi, R. N. V. S.; Sarnaik, H.; Charavaryamath, C.; Rao, B. S.; Raheem, M. A.; Das, J.; Iqbal, J.; Rajagopalan, R. J. Med. Chem. 2002, 45, 3953.

[10] Mallesham, B.; Rajesh, B. M.; Reddy, P. R.; Srinivas, D.; Trehan, S. Org. Lett. 2003, 5, 963.

[11] McNulty, J.; Nair, J. J.; Sliwinski, M.; Harrington, L. E.; Pandey, S. Eur. J. Med. Chem. 2007, 5669.

[12] Adam, W.; Bosio, S. G.; Turro, N. J.; Wolff, B. T. J. Org. Chem. 
2004, 69, 1704.

[13] Friestad, G. K.; Draghici, C.; Soukri, M.; Qin, J. J. Org. Chem. 2005, 70, 6330 .

[14] Yue, X.; Zhang, X.; Qing, F.-L. Org. Lett. 2008, 11, 73.

[15] Masuda, N.; Takahashi, Y.; Otsuki, M.; Ibuki, E.; Miyoshi, H.; Nishino, T. Antimicrob. Agents Chemother. 1996, 40, 1201.

[16] Hoveyda, H. R.; Pinault, J. F. Org. Lett. 2006, 8, 5849.

[17] Ito, H.; Saito, A.; Taguchi, T. Tetrahedron: Asymmetry 1998, 9, 1979.

[18] Cao, Y.; Suzuki, K.; Tajima, T.; Fuchigami, T. Tetrahedron 2005, $61,6854$.

[19] Yang, X. Y.; Fang, X.; Ju, Z. H.; Hu, Y. N.; Wu, F. H. Synthesis 2011, 3627.
[20] Grega, K. C.; Barbachyn, M. R.; Brickner, S. J.; Mizsak, S. A. J. Org. Chem. 1995, 60, 5255.

[21] Huang, W.-Y.; Wang, W.; Huang, B.-N. Acta Chim. Sinica 1985, 43, 409 (in Chinese) (黄维垣, 王巍, 黄炳南, 中国化学学报, 1985, 43, 409.)

[22] Huang, W.-Y.; Wang, W. Acta Chim. Sinica 1986, 44, 940 (in Chinese). (黄维垣, 王巍, 中国化学学报, 1986, 44, 940.)

[23] Bertau, M.; Bürlib, M.; Hungerbühlerc, E.; Wagner, P. Tetrahedron: Asymmetry 2001, 12, 2103.

[24] Agami, C.; Couty, F.; Rabasso, N. Tetrahedron Lett. 2000, 41, 4113.

(Li, L.; Fan, Y.) 